\title{
Recovery and Characterization of an Ancient Bacillus sphaericus from Dominican Amber
}

\author{
RAUL J. CANO\& MONICA K. B ORUCKI*
}

We report the isolation, cultivation, identification, and partial characterization of a Bacillus sphaericus-like bacteria from the abdominal tissue of a 2540 million year old Proplebeia dominicana (Apidae:

Hymenoptera) entombed in Dominican amber. A total of 16 extractions of $P$. dominicana from amber were performed, of which, one yielded bacterial growth from abdominal tissue. Process validation studies indicated that our decontamination procedures sterilized the surface of the amber. No microorganisms grew in any of the controls. Based on biochemical and enzymatic profiles of the putative ancient isolate, as well as on nucleic acid sequences, it appears that the putative ancient Bacillus sp. is most closely related to Bacillus sphaericus. The 165 rRNA gene for the putative ancient Bacillus isolate was amplified, and sequenced. The nucleic acid sequences were used in blast searches of non-redundant nucleic acid data bases and the highest scores were obtained from Bacillus spp. Sequence data derived from the $1482 \mathrm{bp}$ segment of rRNA was used to estimate the substitution rates of the isolate and construct phylogenetic trees. Phylogenetic analyses placed the putative ancient Bacillus sp. in a sister group with extant $B$. sphaericus. Based on phenetic, enzymatic, and molecular studies it appeared that the $B$. sphaericus-like isolate originated from the abdominal tissue of 25-40 million-year-old $P$. dominicana in Dominican amber.

The isolation of ancient microorganisms from various types of materials, including salt crystals, deep earth cores, and fossilized organisms has been reported previously $(1,2)$. As is the case with all claims of ancient microbial isolations, they have been clouded by criticisms of envi- ronmental contamination. Many of these criticisms stem from the fact that extrapolation of survival curves based on radiation damage and macromolecular decay predict that such isolations are not feasible (3).

The possibility that ancient bacterial endospores have survived preserved in amber for millions of years is intriguing and significant for several reasons. Due to the scarcity of bacterial fossils in the geologic record, morphological and biochemical data about ancient bacteria is generally not available, precluding detailed studies of their metabolism, origin and evolution. Sequence data derived from the $16 \mathrm{~S}$ rRNA has been used recently to construct a phylogenetic tree for prokaryotes (4). Sequence data from both ancient bacterial DNA and Bacillus spp. from dated amber samples would help validate or adjust the existing view of bacterial phylogeny and the rate of nucleotide substitution for various genes.

Bacillus is an ancient and ubiquitous bacterial genus capable of forming endospores (5) Endospores are resting spores able to withstand adverse conditions that kill vegetative bacterial cells. The endospore's contents are in a state of virtual dehydration and are stored inside a thick, protective protein coat that affords it resistance to varying degrees of heat, radiation, pressure, and chemical agents (6). The mechanism of resistance to these physical and chemical agents has been studied in detail (7) and include the chemical environment within the spore, enzymatic dormancy, dehydration, conformational changes of the genome. and intrasporal crystalline structure. Resistance to radiation is particularly important to the long term survival of the endospore as it results in damage to the genetic material (6). It appears that the degree of dehydration and the predominance of the condensed form of the DNA molecule (A configuration) reduce significantly the level of radiation damage. Based in these and other observations, it appears that the duration of

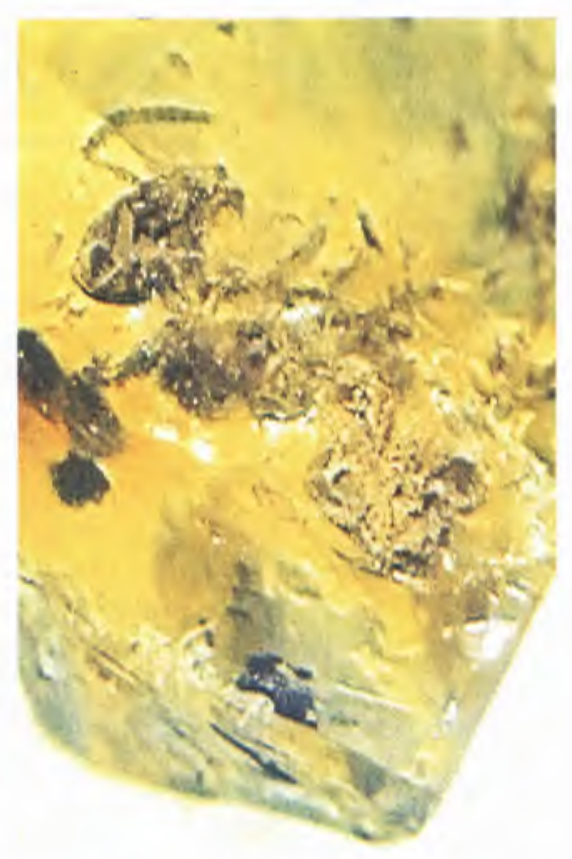

cryptobiosis by the endospore is much longer that previously believed, and might extend into the millions of years.

It is well established that there is a symbiotic relationship between $\mathrm{Ba}$ cillus species and many species of bees $(9-17)$. There is also evidence that this relationship dates back millions of years as Bacillus DNA has been amplified from the abdominal tissue of 25-40 million year old bees that were preserved in amber (18). Furthermore, it is possible that the tissue of these amber-entombed bees may harbor viable Bacillus endospores preserved in a desiccated state for millions of years. The successful germination and culture of these ancient endospores would allow the study of the physiology and may provide insights into ancient symbiotic relationships and host-microbe relationships.

Bacillus spp. have been consistently isolated from the abdominal cavity, glandular secretions, pollen, and larval provisions of stingless bees (e.g., Trigona, Melipona, Plebeia, Centris, and Anthophora) and honey bees (e.g., Apis) and appear to play a vital role in the production, metabolic conversion, and/or preservation of larval provisions. In particular, $B$. subtilis, B. megaterium, B. pumilus, $B$. 
sphaericus, and B. circulans are frequently associated with bees. A study by Gilliam et al. (17) reported a striking similarity between the microbial contents of different types of food from two social bees. They hypothesized that this similarity may reflect similar metabolic roles of $\mathrm{Ba}$ cillus species that have evolved in the nutrition of bees. The results of a study by Machado (19) supported this hypothesis. This study uncovered an association between the pollen of Melipona quadrifasciata, a stingless bee, and a Bacillus species resembling B. pumilus. Machado reported that the elimination of this Bacillus species from pollen stores caused eventual death of the colony.

Proplebeia dominicana (Apidae: Meliponinae) is an extinct species of neotropical, stingless bees found in 25-40 million year old amber from the Dominican Republic (20). P. dominicana is relatively common in Dominican amber presumably because individuals became entrapped when they attempted to collect resin balls for nest construction $(21,22)$. The presence of Bacillus spp. in the abdominal cavity of these bees was first demonstrated by electron microscopy (22) and later by the isolation of Bacillus DNA in the abdomen of 25-40 million year old $P$. dominicana preserved in amber (18).

While the ubiquitous distribution of Bacillus spp. increases the probability of preservation in amber, it also poses problems when trying to ensure that the Bacillus isolates extracted from amber inclusions are of ancient origin rather than extant laboratory contaminants. Endospores are the most resistant living form of life on earth (6) and are therefore resistant to many methods used to disinfect the laboratory work area. Consequently, the area and the equipment used in the extraction procedure were set up with this in mind. It is also important that any unidentified ancient bacterial isolates not be taken out of the confines of the safety cabinet until thoroughly identified and characterized. For these reasons, the extraction and isolation procedures were designed utilizing techniques employed in tissue culture and medical microbiology to develop an extraction method that will both prevent contamination and contain ancient isolates within the confines of the safety cabinet. As an additional precaution, all incubations were carried out in locked incubators and the isolate stored in a locked freezer within a locked room with limited personnel access to the room.

Dominican amber specimens containing Proplebeia dominicana fossils and dating 25-40 million years old, were obtained from Ambergene Corporation, San Francisco, CA. Sixteen extractions of tissues from distinct $P$. dominicana fossils were performed in a decontaminated, class II laminar flow hood. The amber was surface sterilized and cracked as described by Cano et al. (23). Briefly, amber samples were immersed in $2 \%$ buffered glutaraldehyde at room temperature (RT) for 12 hours. The amber pieces were then rinsed three times in sterile double-distilled water (sddw) and immersed in 10\% bleach for an additional 12 hours at RT. This treatment was followed by three rinses of sddw and immersed in $70 \%$ ethanol for 2 hours at RT. The alcohol was evaporated by flaming the amber piece. Trypticase soy broth (TSB) (BBL, Cockeysville, Md.) was inoculated with the bee tissue samples to test for the presence of viable endospores. TSB was also inoculated with $100 \mu \mathrm{l}$ samples of the solutions used in the sterilization process and with pieces of the interior or exterior of the amber. The TSB was incubated aerobically for two weeks at $35^{\circ} \mathrm{C}$. Tubes showing evidence of growth were subcultured onto Trypticase soy agar (TSA) (BBL, Cockeysville, Md.) and identified according to biochemical methods described by Gordon (20). Three plates of TSA were placed in the hood to test for environmental contaminants. The plates were left open throughout the entire extraction procedure. At the end of the procedure, the Petri plate lids were re- placed and the plates were incubated at $35^{\circ} \mathrm{C}$ for two weeks.

An isolate of Bacillus sp. was obtained from the TSB inoculated with $P$. dominicana abdominal tissue and coded as BCA16. No Bacillus spp. were isolated from any of the other TSB tubes inoculated with the solutions used in the sterilization procedure or the interior or exterior of the amber. No contamination was detected on the TSA plates left open in the hood during the extraction procedure.

The effectiveness of the sterilization procedure was tested by inoculating eleven pieces of amber (without indusions) in a TSB an endosporulating culture of Bacillus subtilis. The number of endospores was estimated (by direct count) to be $1.6 \times 10^{7}$ endospores/ml of TSB. The amber was allowed to soak overnight in the culture before carrying out the sterilization procedure. Tubes containing 10 $\mathrm{ml}$ TSB were inoculated with the surface-sterilized amber and incubated at $35^{\circ} \mathrm{C}$ for 14 days. No bacterial growth was detected in any of the eleven TSB tubes after the two-week incubation period.

"Mock extractions" were also performed to check for environmental contamination of the samples during the extraction procedure. Two pieces of amber (without inclusions) were surface-sterilized and the extraction procedure was simulated inside a laminar flow hood. The amber was cracked using liquid nitrogen, and manipulated under a disinfected dissecting microscope using sterile tweezers and needles for approximately 45 minutes. Samples of the solutions used in the sterilization procedure and from both the interior and exterior of the amber were placed in TSB and incubated at $35{ }^{\circ} \mathrm{C}$ for 14 days to test for the presence of Bacillus endospores. No bacterial isolates were obtained from any of the mock extractions.

The putative ancient Bacillus isolate (BCA16) was identified as $B$. sphaericus by conventional methods (24) and evaluated as to its enzymatic repertoire using the API-ZYM ${ }^{\circledR}$ 
Table 1. Enzymes produced by Bacillus sphaericus soil and bee-derived solates. using the API-ZYM system.

\begin{tabular}{|c|c|c|c|c|c|c|}
\hline Ensyme & NM131 & PJ231 & PJ181 & $\begin{array}{l}\text { ATCC } \\
138052\end{array}$ & $\begin{array}{l}\text { ATCC } \\
179322\end{array}$ & BCA 163 \\
\hline Control & 0 & 0 & 0 & 0 & 0 & 0 \\
\hline Alkaline Phosphatase & 1 & 1 & 1 & 0 & 0 & 0 \\
\hline Butyrate esterase & 4 & 3 & 3 & 1 & 1 & 3 \\
\hline Caprylate esterase lipase & 3 & 3 & 3 & 1 & 1 & 3 \\
\hline Myristate lipase & 1 & 0 & 1 & 1 & 0 & 0 \\
\hline Leucine aminopeptidase & 1 & 1 & 0 & 2 & 2 & 0 \\
\hline Valine aminopeptidase & 0 & 1 & 1 & 1 & 0 & 0 \\
\hline Cystine aminopeptidase & 0 & 0 & 0 & 1 & 0 & 0 \\
\hline Trypsin & 0 & 0 & 0 & 0 & 0 & 0 \\
\hline Chymotrypsin & 2 & 3 & 1 & 0 & 1 & 0 \\
\hline Acid phosphatase & 0 & 1 & 0 & 0 & 0 & 0 \\
\hline Phosphoamidase & 0 & 0 & 0 & 0 & 0 & 2 \\
\hline$\alpha$-galactosidase & 0 & 0 & 0 & 0 & 0 & 5 \\
\hline$\beta$-galactosidase & 1 & 0 & 1 & 0 & 0 & 2 \\
\hline$\beta$-glucuronidase & 0 & 0 & 0 & 0 & 0 & 0 \\
\hline$\alpha$-glucosidase & 0 & 1 & 0 & 4 & 0 & 0 \\
\hline$\beta$-glucosidase & 1 & 0 & 1 & 0 & 0 & 2 \\
\hline $\mathrm{N}$-acetyl- $\beta$-glucosaminidase & 0 & 0 & 0 & 0 & 0 & 0 \\
\hline$\alpha$-mannosidase & 0 & 0 & 0 & 0 & 0 & 0 \\
\hline$\alpha$-fucosidase & 0 & & 0 & 0 & 0 & 0 \\
\hline
\end{tabular}

1. Soil isolates

2. Stingless bee-derived isolates

$3.0=<10 \mathrm{~nm}$ enzyme; $1=10 \mathrm{~nm}$ enzyme; $2=20 \mathrm{~nm}$ enzyme; $3=30 \mathrm{~nm}$ enzyme; $4=40 \mathrm{~nm}$ enzyme $5 \geq 50 \mathrm{~nm}$ enzyme.

system (Analytab Products Inc., Plainview, New York) according to the manufacturer's instructions. The enzymatic profiles obtained by using the APIZYM ${ }^{\circledR}$ tests are shown in Table 1. The enzymatic profiles of the putative ancient Bacillus sp. isolated from Proplebeia dominicana compared well with enzymatic profiles of B. sphaericus isolates from extant bees.

Gilliam (12-16) reported that the lipases most often associated with tropical bees are caprylate esterase lipase and butyrate esterase (Table 1). These were also produced by BCA16. Neither the bee associated Bacillus spp. tested nor BCA 16 produced cystine aminopeptidase, $\beta$-glucuronidase, $\mathrm{N}$-acetyl- $\beta$-glucosaminidase, $\alpha$-mannosidase, and $\alpha$ fucosidase. The putative ancient $B a$ cillus sphaericus isolate (BCA 16), however, tended to produce more butyrate esterase and caprylate esterase lipase than did the extant soil isolates of B. sphaericus tested. Ex- tant B. sphaericus isolates derived from stingless bees produced approximately the same levels of the above mentioned enzymes as BCA 16. Extant bee-derived isolates of B. sphaericus generally produced higher levels of chymotrypsin and alkaline phosphatase than did BCA16. BCAl6, on the other hand, produced four enzymes not produced by any of the extant strain of $B$. sphaericus. The enzymes produced were phosphoaminidase, $\alpha$-galactosidase, $\beta$-galactosidase, and $\beta$-glucosidase. These data seem to support the hypothesis that BCAl6 originated from the abdominal tissue of the fossil bee Proplebeia dominicana rather than contamination from soil- or bee derived extant B. sphaericus.

The biochemical identification and enzymatic data gathered in this study cannot refute the hypothesis that viable Bacillus endospores can be recovered from the 25-40 million year old abdominal tissues of Prople- beia dominicana entombed in amber. The bacterium isolated from fossilized bee tissue (i) formed endospores, (ii) is a known endosymbiont of bees, and (iii) has an enzyme profile similar to that of Bacillus species found in the abdomen of extant bees.

The presence of Bacillus DNA was evaluated in each of the 16 extractions. DNA was extracted from samples of abdominal tissue of $P$. dominicana, the solutions used in the sterilization procedure, and small pieces of the interior and exterior of the amber. DNA extractions were performed using Glassmill ® SpinBuffer from an RPMTM ${ }^{\mathrm{TM}}$ kit (Bio 101, La Jolla, CA) as described by Cano and Poinar (25). A 530 basepair (bp) fragment of the 16S rDNA was amplified by polymerase chain reaction (PCR) using primers specific for Bacillus spp. BCA34IF/BCA871R18 in 6 out of 16 amplifications of abdominal tissues assayed. No amplified DNA was obtained from the soluti- 
ons used in the sterilization procedure or from the interior or exterior of the amber. One of the sequences, PD_EX06, obtained from a fossilized bee tissue in the same amber specimen from which BCAl 6 was isolated, was very similar to the sequence of BCA16 (Figs. $1 \&$ 2).

DNA was also extracted from BCA and amplified by PCR using the 16 srRNA primers of Edwards et al (25). The resulting 1482 bp amplicon was by cycle sequecing using fluorescent dideoxy terminators (PerkinElmer, Applied Biosystems Division, Foster City, CA). Sequences were obtained a Gene Sequencer 373 (Perkin-Elmer, Applied Biosystems Division, Foster City, CA) and aligned with the aid of CLUSTAL using the Genetic Data Environment version 2.2 (Steve Smith, personal communication) and verified visually.

The 16S rDNA nucleotide sequences of BCA16 and PD_EX06 were compared to homologous sequences of Bacillus spp. listed in GenBank. Lactobacillus casei (accession number X61165) was used as the outgroup. Phylogenetic trees were constructed using Maximum Likelihood (DNAML) algorithms of Phylip 3.5 (26). The maximum likelihood tree is illustrated in Fig. 1. The tree shows BCA16 and PD_EX06 in a sister group, and both of which in a sister group with $B$. sphaericus. It is noteworthy that BCA16, PD_EX06, and $B$. sphaericus were grouped by the algorithm with Sporosarcina ureae, the only other round-spored endospore former (Group 2) analyzed. Phylogenetic trees were also constructed using eight B. sphaericus sequences obtained from GenBank (Fig. 2) as well as BCAl6 and PD_EX06. The consensus tree from 2,000 replicate trees using DNAML placed both, BCAl6 and PD_Ex06 in the same sister group and ancestral to all other $B$. sphaericus isolates except B.SPHAER4.

The rate of nucleotide substitution, $r$, was calculated by dividing the number of substitutions between the putative ancient $P$. dominicana isolate and extant B. sphaericus by the number of nucleotide sites analyzed

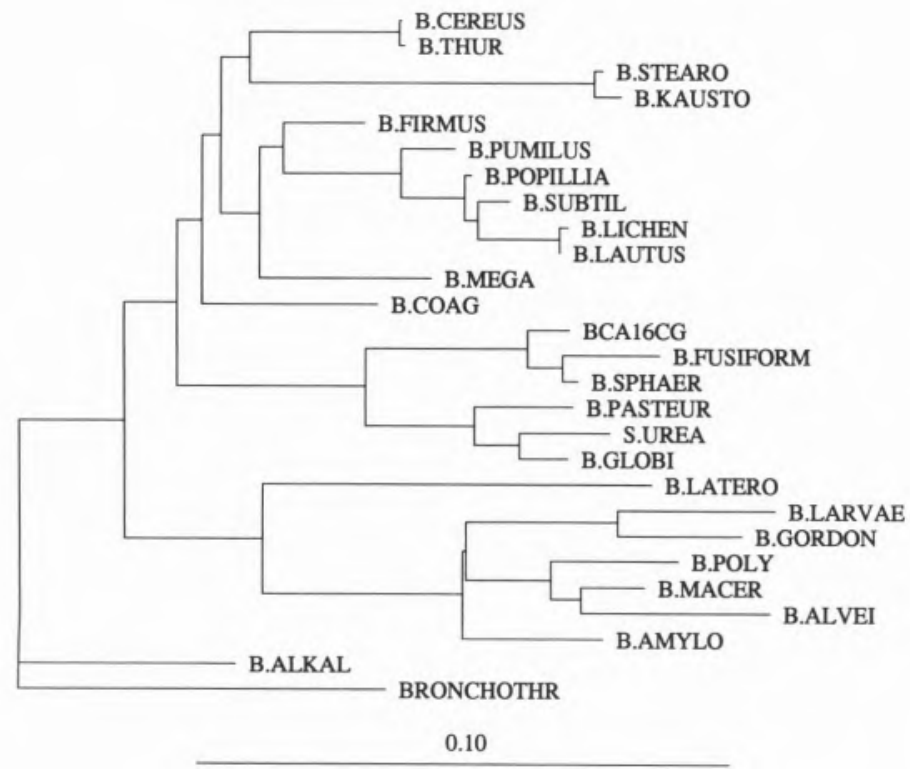

Fig. 1 - Evolutionary tree for the Bacillus spp. analyzed. Trees were constructed by the Maximum Likelihood method using the DNAML program of PHYLIP 3.5 (26) and a least squares algorithm for fitting additive trees to proximity data (33). Bronchothrix campestris (X56156). was used as the outgroup, with 2,000 bootstrap replications, randomized data input and global rearrangement of data. A total of 6 independent runs were evaluated. All resulting trees were identical. Branch lengths were drawn to scale, using branch lengths obtained from Maximum Likelihood analysis and TreeTool (34).

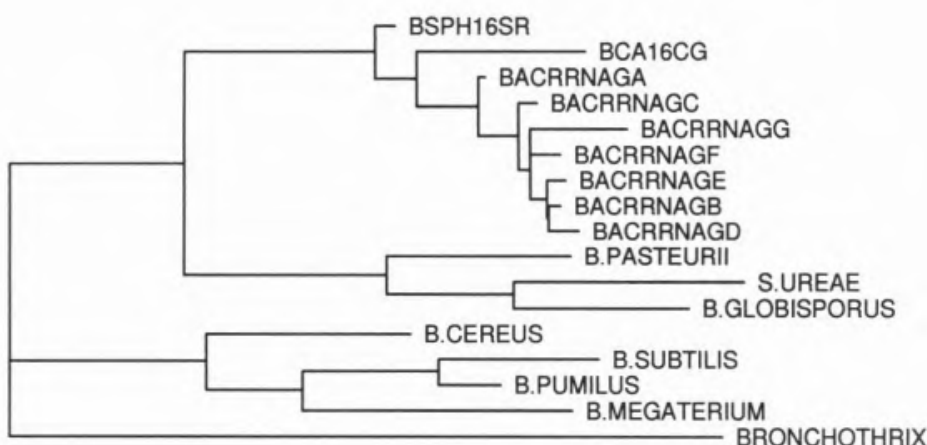

0.10

Fig. 2 - Phylogenetic tree for Bacillus sphaericus. Accession numbers Bacillus sphaericus are as follows: BACRRNAGF (L14015); BACRRNAGD (L14013); BACRRNAGE (L14014); BACRRNAGB (L14011); BACRRNAGC (L14012); BACRRNAGA (L14010); BACRRNAGG (L14016); BSPH16SR (X60639); S. ureae, (138654); B. pasteurii (X60631); B. globisporus (X60644); B. cereus (X55063); B. subhlis (X60646); B. pumilus (X60637); B. megaterium (X60629); and BCA16CG, the putatively ancient B. sphaericus isolate BCA16 (L38654). Sequences were aligned manually using the Genetic Data Environment (GDE 2.1) text editor. Trees were constructed by the Maximum Likelihood method using the DNAML program of PHYLIP 3.5 (17) and a least squares algorithm for fitting additive trees to proximity data (33). Bronchothrix campestris (X56156). was used as the outgroup, with 2,000 bootstrap replications, randomized data input and global rearrangement of data. A total of 6 independent runs were evaluated. All resulting trees were identical. Branch lengths were drawn to scale, using branch lengths obtained from Maximum Likelihood analysis and TreeTool (34). 
(27). This number, $K$, was then divided by $2 T$, where $r$ is the time of divergence between the two sequences. T can be estimated at 25-40 million years based on the age of the Dominican amber used in the extraction. The nucleotide substitution rate was calculated as $0.3-0.7 \times 10^{9}$ substitutions per site per year.

Phylogenetic studies showed that the 16S rDNA of the ancient isolate was similar to both amplicons from fossilized abdominal $P$. dominicana tissues, and similar, but not identical, to that of extant Bacillus sphaericus (Fig. 2). The nucleotide substitution rate estimated was slightly greater than those reported previously for bacteria. Ochman and Wilson (28) estimated the nucleotide substitution rate of the eubacterial 16S rRNA at $0.1 \times 10^{-9}$ substitutions per site per year while Moran et al. (29) estimated the nucleotide substitution rate of $16 \mathrm{~S}$ rRNA of aphid symbionts at 0.1-0.3 $\times 10^{-9}$ substitutions per site per year.

The discrepancies in nucleotide substitution rates may have been due to the method at determining substitution rates. Ochman and Wilson (28) estimated divergence time by tying ecological events that took place at known times in the geological past to specific branch points in the genealogical tree relating the $16 \mathrm{~S}$ ribosomal RNAs of eubacteria, mitochondria, and chloroplasts. Moran et al. (29), reconstructed the phylogenetic trees of the endosymbiotic bacteria using the phylogenetic trees of their aphid host (31). The method we used for arriving at our estimation of base substitution rates was based on the age of the amber fossil from which the presumed ancient $B$. sphaericus was isolated (25-40 million-years before present).

Despite the discrepancy in the rate of nucleotide subsfftutions, it appears that the presumed ancient $B$. sphaericus shows more nucleotide substitutions when compared to extant B. sphaericus than extant $B$. sphaericus isolates among themselves. It is noteworthy that the sequence of the PCR product from enzymatic amplification of fossilized $P$. dominicana abdominal tissues (PD_EX06) most closely resembled that of the $B$. sphaericus isolate from $P$. dominicana rather than those of extant strains of $B$. sphaericus. This observation further seems to indicate that the B. sphaericus -like isolate from abdominal tissue of $P$. dominicana is of ancient origin rather than an environmental contaminant.

In the absence of extensive population genetic data for extant organisms, which could test for aberrant rates of substitution that would support the antiquity of the sample, the correct approach to test the assertion of an ancient origin would be to hypothesize that the isolate BCA16 is a laboratory contaminant. As B. sphaericus can be readily cultured from natural environments, it should not prove difficult to isolate it from the laboratory environment if it is present therein. As an integral part of our experiments, we monitor the level and nature of microbial contamination in and around the laboratory environment in an ever-increasing geographical radius, with the assumption that contamination will have the greatest chance of occurring from the sample, then from the safety cabinet in which the amber is processed, then from the laboratory at large, then from the building, and finally from the grounds surrounding the building. We have processed more than 80 amber specimens, both prior to and after the recovery of BCA16. During those recoveries we have sampled the sterilized amber itself prior to processing as well as representative sites of the safety cabinet, the laboratory, and the building itself (including the filters on air ducts). We have cataloged potential contaminants on the surface of Dominican and Mexican ambers, these include coliforms, diphtheroids, pseudomonads, endosporeforming rods, and other Gram positive bacteria, but not $B$. sphaericus. Similarly, we have not recovered $B$. sphaericus from any of the sites tested to date. Based on these results, the only plausible source of BCA 16, we have concluded, is from within the amber inclusion (Proplebeia dominicana) itself. We continue to sample the laboratory environment for B. sphaericus in order to further test the hypothesis.

We fully recognize there is no means available to absolutely prove that BCA16 is indeed of ancient origin as opposed to an environmental contaminant. Our process validation studies, however, showed that the chemical treatment to which the amber specimens were subjected, indeed sterilized the surface of the amber. In addition, the environmental contamination controls were repeatedly negative for growth, not only of Bacillus spp. but of all microorganisms, placing a greater degree of reliability on the aseptic techniques employed in the extraction procedures of fossilized bee tissues. As the room and the safety cabinets therein are only used for the isolation of ancient materials and never extant bees, the possibility that BCAl6 is a product of extant, beederived Bacillus contamination is also diminished. Biochemical analyses seem to indicated that the biochemical profile of the putative ancient bacterium resembles that of extant bee-isolated B. sphaericus, but sufficiently different from extant isolates tested to obviate the possibility of contamination from these isolates. Finally, the nucleotide substitution rate of the presumed ancient bacterial isolate is consistent with the claim of antiquity of the isolate. Based on the results described above, it appears that the $B$. sphaericus -like organism isolated from $P$. dominicana entombed in amber is indeed of ancient origin.

The ancient origin of BCA16 (or any other isolate from amber) will not be established by any single test, but from the accumulation of results using different approaches which would make the hypothesis that BCA16 originated from laboratory contamination implausible. We have used several lines of evidence to evaluate the ancient isolate with modern counterparts. We believe, also, 
that we have eliminated all the obvious causes of contamination as sources of BCAl6. We are currently exploring less obvious sources of contamination, induding the penetration of environmental, extant bacteria into the amber inclusions through their secretion of enzymes that digest the amber matrix, and/or through microfissures that have subsequently closed. We have also considered the suggestion that BCAl6 is a "modern" isolate that had been multiplying within the bee's abdominal cavity since its entrapment in the resin. This latter hypothesis is also implausible as B. sphaericus is strictly aerobic and would soon consume all the oxygen available for their growth. Furthermore, even if a single cell could multiply at a rate of 1 generation every 200,000 years, after 20 million years, they would have undergone 100 doublings, yielding approximately $1 \times 10^{30}$ cells. This is clearly impossible. Although further tests must be done, when all the evidence gathered thus far are evaluated and weighed, they appear to support our claim that BCA16 is indeed ancient. The prospect that viable microorganisms can be recovered from amber inclusions and grown in the laboratory is an exciting one. These microorganisms can serve as tools to study evolutionary processes and genome structure and function as well as sources of novel enzymes and secondary metabolites. An essential prerequisite to scientific exploitation of such organisms, though, is critical authentication of their ancient origin. As such, critical reviews of our work are both necessary and indeed welcome. To realize progress from such healthy skepticism, independent investigators must conduct their own experiments or offer alternative means to verify or refute the hypothesis that these microorganisms are of ancient origin.

\footnotetext{
* Biological Sciences, California Polytechnic State University, San Luis Obispo, CA, 93407 USA Keywords: amber, Bacillus, endospore-former. fossil paleontology, Proplebeia dominicana
}

\section{REFERENCE}

1. M. Kennedy, S. L. Reader, L. M. Swierczynski. Microbio$\log y 140$ (1994) 2513

2. M. R. D. Seaward, T. Cross,. B. A. Unsworth. Nature 261 (1976) $407-408$.

\section{T. Lindahl, Nature 362 (1993) 709}

4. C. R. Woese. The Use of Ribosomal RNA in Reconstructing Evolutionary Relationships among Bacteria. Evolution at the Molecular Level. R. K. Selander, A. G. Clark, T. S. Whittan,: Sinauer Associates, Inc., (1991).

5. D. Claus and R.C. W. Berkeley, "Genus Bacillus". Bergey's Manual of Systematic Bacteriology. Ed. P. A. Sneath. Baltimore: Williams and Wilkins, (1986).

6. P. I. Setlow. Appl. Bacteriol. Symp. Suppl. 76 (1993) 495 605 .

\section{Ann Rev. Microbiol. 42 (1988) 319-338}

8. H. Gest and I. Mandelshm. Microbiol. Sci. 4 (1987) 69. 71

9. M. Gilliam and H. L. Morton. Apidologie 9 (1978) 21-22

10. B. J. Lorenz, G. V. Richardson. Microbios 55 (1988) 95 114

11. D. W. Roubik, B, J. Lorenz. Apidologie, (1990) 89-97

12.? and D. K. Valentine, I. Invert. Pathol. 28 (1976) 275 276

13. ?, S. L. Buchmann, B. J. Lorenz. Apidologie 15 (1984) 1

14. ? and S. Taber m. I. Invert. Pathol. 58 (1991) 286-289.

15. S. L. Buchmann, B. I. Lorenz. Biotropica 17 (1985) 28-31.

16. ?. S. L. Buchmann, B. J. Lorenz, R. I. Schmalzel. Apido logie 21 (1990) 99-105.

17. Apidologie 10 (1979) 269-74

18. R. I. Cano, M. Borucki M. Schweitzer, H. Poinar, G. O Poinar, K. Pollard, Appl. Environ. Microbiol. 60 (1994) 2164-2167

19. I. O. Machado. Ciência e Cultura (São Paulo) 23 (1971 625.633

20. A. Wille and L. C. Chandler. "A New Stingless Bee from Tertiary Amber of the Dominican Republic." Revista Biolo gia Tropical 12 (1964) 187-95.

21. D. W. Roubik. Ecology and Natural History of Tropical Bees. New York: Cambridge Press, 1989.

22. G. O. Poinar, Jr. Experientia 50 (1494) 536-542.

23. R. J. Cano, H. N. Poinar, N. J. Pieniazek, A. Acra, G. O Poinar Ir. Nature 363 (1993) 536-538.

24. R. E. Gordon, W. C. Haynes, C. H-N. Pang. The Genus Bacillus. Washington D. C.: United States Department of Agriculture, (1973).

25. R. J. Cano and H. N. Poinar. Biotechniques 15 (1993) 811. U. Edwards, T. Rogall, H. Blocker, M. Emde, M. and E. Bottger. Nudeic Acids Res. 17 (1989) 7843.

26. J. Felsenstein. Cladistics 5 (1989) 164-166
27. W-H. Li and D. Grauer. Fundamentals of Molecular Evolution. Sunderland, Massachusetts: Sinauer Associates, Inc. (1991).

28. H. Ochman and A.C. Wilson. I. Mol. Evol. 26 (1987) 74 86

29. N. A. Moran, M. A. Munson, P. Baumann, H. Ishikawa. Proc. R Soc. Lond. B. 253 (1993) 167-171

30. M. Gouy and L. Wen-Hsiung. Evolutionary Relationships among Primary Lineages of Life Inferred from rRNA Sequences. The Ribosome. W. E. Hill, P. B. Moore, A. Dahlberg, D. Schlessinger, R. A. Garrett, J. R. Warner, eds. Washington D. C.: American Society for Microbiology, (1990).

31. It is also possible that the relatively large number of substitutions seen in the putative ancient $B$. sphaericus $16 \mathrm{~s}$ rRNA was due to post-germination repair of accumulated damage over the 25-40 million-year cryptobiotic state.

32. DNA was extracted using a silica gel suspension as described by Cano and Poinar (see ref. 25). For PCR amplification, $5 \mu \mathrm{l}$ of extracted DNA was used as the source of DNA for enzymatic amplification by PCR. Symmetric PCR amplifications were performed using 2 units of low DNA AmpliTaq DNA polymerase (Perkin-Elmer, Norwalk, CT) $2 \mu \mathrm{g} / \mathrm{ml}$ bovine serum albumin, $0.5 \mu \mathrm{M}$ each of $\mathrm{BCA} 341 \mathrm{~F}$ and $\mathrm{BCA871R}$ primers, $2.0 \mathrm{mM} \mathrm{MgCl}$, and 0.2 $\mathrm{mM}$ deoxynucleotide triphosphates in a total volume of 50 $\mu \mathrm{l}$. All reagent mixtures and sample dilutions were performed in an ice water bath and the tubes placed in the ther mal cycler after the heat block reached $80^{\circ} \mathrm{C}$. Polymerase chain reactions were performed using a Temp-Tronic(i) thermal cycler dry bath (Thermolyne, Dubuque, IA). Du ring the first cycle the DNA templates were denatured for 2 min at $95{ }^{\circ} \mathrm{C}$ followed by a primer armealing step at $58^{\circ} \mathrm{C}$ for $1 \mathrm{~min}$, and an extension step of $1 \mathrm{~min}$ at $72{ }^{\circ} \mathrm{C}$. The following 30 cycles consisted of a 1 min denaturation step at $94^{\circ} \mathrm{C}$, a $1 \mathrm{~min}$ primer annealing step at $58{ }^{\circ} \mathrm{C}$, and a 1 min primer extension step at $72{ }^{\circ} \mathrm{C}$. The last cycle consisted of a 1 min denaturation step, a 1 min primer an nealing step, and a 10 min primer extension step. The products of each amplification were evaluated by $1.2 \%$ agarose in Tris-Acetate-EDTA buffer. Amplification products were cloned using a TA Cloning@ kit (Invitrogen, San Diego, (CA) as per manufacturer's instructions. Cloned plasmid DNA was sequenced using a Sequenase II DNA sequencing kit (USB, Cleveland, $\mathrm{OH}$ ) with $\alpha$-thio $35 \mathrm{SdATP}$ as per manufacturer's instructions. Three clones were sequenced for each sample, using both $\mathrm{SP} 6$ and $\mathrm{T}$ ? sequencing primers. Electrophoresis of sequencing products was performed in a $6 \%$ sequencing gel (Gel-Mix 6 Gibco BRL, Gaithersburg, MD). Autoradiographs of airdried sequencing gels were made using XAR X-ray film (Kodak, Rochester, MN). Sequences were scanned into a Spara 10 station (Sun Microsystems, Foster City, CA) and analyzed using the Biolmage DNA analysis software (Millipore, Bedford, MA). Autoradiographs were evaluated manually to verify the computer-analyzed sequences. Sequences were aligned manually using the Genetic Data Environment (GDE 2.1) text editor.

33. G. De Soete, Psychometrika 48 (1983) 621-626.

34. M. Maciukenas, Ribosomal Database Project.

35. We wish to thank Dr. Martha Gilliam for her invaluable advice on bee-Bacillus symbiotic relationships and Dr. David W. Roubik for providing the stingless bees used in this study. We also wish to thank our technician Dawn Marie Norton for her insatiable need to meet her deadlines with respect to this study. Funds for this study were kindly provided by Ambergene Corporation, San Carlos. CA, USA. 